\title{
I TEMPI DEL PASSATO
}

0. Normalmente in italiano, in una frase principale, per riferirci al passato, abbiamo la scelta fra Imperfetto (scrivevo), Passato Prossimo (PP) (ho scritto), e Passato Remoto (PR) (scrissi). Trascuriamo qui il fatto che Imperfetto e PR appartengano inequivocabilmente al paradigma morfologico del verbo, mentre il PP è una forma composta, costruita sintatticamente, che si potrebbe voler affiancare ad altri costrutti, come sto scrivendo ecc.; del resto ci sono proposte generativiste di considerare il PP come risultante dall'inserimento di una frase al passato entro un predicato al presente (l'ausiliare). Postuliamo invece che, a qualche livello, si ponga, nel sistema verbale italiano, la scelta fra i tre paradigmi citati (indipendentemente dalla constatazione che essi di fatto compaiono nelle tradizionali grammatiche italiane). Notiamo anche che il Trapassato Prossimo, in maniera esplicita o implicita, e il Trapassato Remoto in maniera esplicita, nell'italiano moderno indicano anteriorità relativa, rispetto a un altro punto di riferimento passato.

Se cerchiamo di enunciare le modalità d'uso di questi tre paradigmi (Imperfetto, PP e PR), ci scontriamo con notevoli difficoltà.

1. La grammatica tradizionale suddivide il sistema verbale in base alle due grandi categorie dei modi e dei tempi.

I modi, si dice di solito, hanno a che vedere col 'grado di realtà' dell'azione; oppure, riferendosi alla struttura sintattica delle frasi: l'indicativo è il modo tipico della reggente, e il congiuntivo, della dipendente.

All'interno dell'indicativo troviamo dei tempi cosiddetti assoluti, che si riferiscono al presente, al passato, e al futuro, cioè alla contemporaneità, anteriorità, e posteriorità rispetto al momento dell'enunciazione; e, come abbiamo detto sopra, dei tempi cosiddetti relativi, che esprimono l'anteriorità rispetto non al momento dell'enunciazione, ma a quello di altri eventi.

Reichenbach (1947) ha formulato efficacemente la distinzione fra (a) punto del discorso o dell'enunciazione, (b) punto dell'evento, e (c) punto di riferimento (una specie di punto prospettico da cui si considera l'evento). Nel Presente (Mario scrive una lettera) tutti e tre i punti coincidono, e nel Trapassato Prossimo (Mario aveva scrito una lettera) sono tuti e tre separati: nel PP (Mario ha scritto una lettera) il punto dell'enunciazione (a) e il punto di riferimento (c) coincidono, e sono posteriori al punto dell'evento (b); nel PR (Mario scrisse una lettera) il punto dell'evento (b) 
e il punto di riferimento (c) coincidono, e sono anteriori al punto dell'enunciazione (a).

Ma è chiaro che la distinzione fra Imperfetto, PP e PR non è relativa soltanto al tempo, bensì anche a qualche altro elemento. Una terza categoria, corrente nella descrizione del sistema verbale di varie lingue, è quella dell'aspetto. Come è noto, in russo, il verbo ha una doppia coniugazione (con i modi e i tempi opportuni): una per l'imperfettivo, che presenta l'azione nel suo svolgersi, e una per il perfettivo, che presenta l'azione in quanto compiuta. Per dire scrivere in russo bisogna scegliere fra due verbi diversi, l'imperfettivo pisat' ("scrivere" come azione in corso di svolgimento), e il perfettivo napisat" ("scrivere" come azione compiuta).

Una distinzione aspettuale simile, ma triplice invece che duplice, è familiare a chi ha studiato il greco classico, con l'opposizione di Imperfetto (égraphon "scrivevo"), Perfetto (gégrapha "scrissi e finii di scrivere"), e Aoristo (égrapsa "scrissi", con riferimento all'atto in sè, senza riguardo al suo svolgimento o compimento). In latino troviamo l'Imperfetto (scribebam, con valore corrispondente a quello dell'Imperfetto greco), e il Perfetto (scripsi con valore corrispondente a quello sia del Perfetto sia dell'Aoristo greco). In italiano, come abbiamo visto, si trovano tre forme per il passato, i cui valori sono diversi da quelli citati per il greco e per il latino.

2. Potrà interessare una parentesi terminologica (per cui si veda Dietrich 1973, pp. 117-119; Knobloch 1961-1986). Aspetto è la traduzione del termine russo vid e si trova nella versione francese di $\mathrm{Ch}$.Ph. Reiff della grammatica russa di N.I. Greč (Gretsch 1828, vol. I, p. 251: aspect); altri termini sono 'gradi' (degrees in Reiff 1857, p. 86), e 'rami' (branches, in francese in Reiff 1851, p. 110, e in inglese in NestorSchnurmann 1884, p. 97).

Vid corrisponde etimologicamente al greco eîdos, che in Dionisio Trace era usato per la distinzione di verbi semplici e derivati. Di fatto, nella grammatica slava di $\mathrm{M}$. G. Smotrickij, all'inizio del Seicento, vid si riferisce alla differenza fra verbi primari e derivati (incoativi e iterativi). Non si tratta ancora della distinzione aspettuale. Questa emerge chiaramente, nella riflessione grammaticale, nel primo Ottocento, con J.S. Vater.

Un altro termine diffuso, a volte come sinonimo di 'aspetto', a volte per indicare una nozione distinta, è quello di Aktionsart (modo, genere, carattere o tipo dell'azione, o, come propone Bertinetto (1986, p. 83), 'azione verbale'). G. Curtius aveva distinto per il greco fra Zeitstufe 'grado temporale', e Zeitart o 'modo o categoria temporale', con riferimento a una azione (Handlung) duratura, momentanea, o compiuta. Brugmann ha usato, per questa nozione, il termine Aktionsart (actio verbi), che è poi stato in seguito spesso usato riguardo al valore lessicale del tema verbale, riservando 'aspetto' alla distinzione grammaticale.

Converrà dunque tenere separata una distinzione come quella di verbi momentanei e durativi da quella dell'aspetto perfettivo e imperfettivo (Šabršula 1961, p. 149): 
a verbi durativi come vivere potrà corrispondere in russo oltre all'imperfettivo proživat' anche il perfettivo prožit'; così a verbi momentanei come morire potrà corrispondere oltre al perfettivo umeret' anche l'imperfettivo umirat'.

Nella tradizione grammaticale greca, Dionisio Trace distingue tre khrónoi (tempi): enestōs (presente), parelèluthōs (passato), e méllōn (futuro); e per il passato introduce quattro distinzioni (diaphorấ): paratatikós (l'Imperfetto), parakeímenos (il Perfetto), hupersuntélikos (il Piuccheperfetto), aóristos (l'Aoristo). La nozione di aspetto, che qui è subordinata a quella di tempo, emerge invece, a quanto pare, con la suddivisione stoica fra tempi hōrisménoi (definiti) e aóristoi (indefiniti), e per i definiti con la suddivisione fra paratatikós (durativo) e suntelikós (compiuto). La suddivisione temporale è secondaria rispetto a quella aspettuale. Secondo M. Pohlenz, Zenone avrebbe usato gli aspetti come criterio di classificazione in quanto era predisposto a metterli in rilievo dalla sua origine semitica. Come è noto il verbo semitico presenta in origine distinzioni aspettuali più che temporali. Anche in Varrone troviamo una distinzione fondamentale fra infectum e perfectum, e solo all'interno di questa troviamo le distinzioni temporali di presente, passato e futuro (scribo, scribebam, scribam, per l'infectum, e scripsi, scripseram, scripsero per il perfectum) (Holt 1943, p. 4; Matthews 1990, pp. 238-239).

3. Tornando ai tempi italiani, una prima distinzione che si può fare, riprendendo celebri saggi di Benveniste e di Weinrich, è quella che contrappone da un lato il Presente e il PP, come tempi commentativi, usati nel discorso, cioè nel dialogo, fra $i$ due attori dell'atto comunicativo (la prima e la seconda persona), e dall'altro l'Imperfetto e il PR, come tempi narrativi, usati nel racconto, o nella storia (riguardante tipicamente la terza persona, un non-attore estraneo all'atto comunicativo). Da questo punto di vista il PP è stato collegato a una prospettiva soggettivizzante, che avvicina l'azione a chi la enuncia, e il PR a una prospettiva oggettivizzante, che da lui la allontana.

4. Una seconda distinzione, relativa alla dinamica narrativa, è quella fra Imperfetto, che dà informazioni di sfondo (Mario continuava a star seduto), e ci lascia in attesa che succeda qualcosa, e il PP o il PR, che presentano un evento che fa procedere la narrativa (si sentirono le prime fucilate). Si tratta, ovviamente, di punto di vista narrativo, e non di caratteristiche obiettive degli eventi. Basta cambiare i tempi perché le fucilate diventino sfondo, e lo star seduto diventi l'evento: si sentivano le prime fucilate: Mario continuò a star seduto.

5. Ci sono certe espressioni che sembrano prestarsi particolarmente all'uso dell'Imperfetto, a quanto pare in relazione al loro significato, generalmente stativo. Le grammatiche parlano a volte in questi casi di imperfetto descrittivo: il cielo era limpido. Anna aveva $i$ capelli scuri. Stammerjohann (1970, p. 333) nota che nel suo 
corpus di testi toscani di tipo parlato si ha prevalenza di Imperfetti con verbi come essere, avere, stare, volere, potere, dovere, e di PR con verbi come dire, fare, andare, venire, vedere, mettere, prendere. Ci si può chiedere se non si tratti di un caso particolare del paragrafo precedente, cioè di espressioni che, per il loro significato, di preferenza vengono usate, rispettivamente, o come sfondo o come evento, nella narrativa. In questo uso rientrano forse anche le designazioni delle date e dell'ora: era il 3 maggio, erano le due e 25. In un testo come: Guardò l'ora. Erano le due e 25. Decise di telefonare, si può pensare che $\mathrm{i}$ due $\mathrm{PR}$ indichino eventi che fanno procedere la narrativa, e che l'Imperfetto appartenga allo sfondo (come un'informazione che il narratore dà direttamente al lettore); oppure che esso si inserisca nel tessuto narrativo attraverso un presupposto vide che (erano le due e 25) (in stile indiretto libero, ci verrebbe allora detto ciò che il personaggio pensa guardando l'orologio, o comunque l'informazione ci viene data dal suo punto di vista).

6. Esistono ovviamente altri valori che questi paradigmi possono assumere. Per esempio l'Imperfetto può designare un'azionè ripetuta, abituale: scriveva delle lettere gentili. Ma se tale azione è situata in un periodo compiuto, l'Imperfetto è impossibile, e si riccore al PR: Per un anno scrisse delle lettere gentili. E così via, dall'Imperfetto narrativo, che si diffonde dall'inizio dell'Ottocento, a tanti altri usi a cui non accenniamo qui.

7. Dal punto di vista dell'aspetto si tratta di opposizione fra l'imperfettività dell'Imperfetto, per cui l'azione è concepita nel suo svolgimento, a prescindere dal suo inizio e dalla sua fíne, e la perfettività del PP e PR, per cui l'azione è concepita nella sua totalità, globalmente, come una singola unità, entro la quale possono acquistare rilievo, nel PP, il momento finale (aspetto di compiutezza), e nel PR una considerazione puntuale (aspetto aoristico), per cui l'azione è presentata in sè, indipendentemente dalla sua durata; il PR può anche mettere in luce il momento iniziale dell'azione, e avrà allora un valore ingressivo. Su questo argomento si veda Bertinetto, 1986, p. 19 segg.

Cerchiamo di vedere come si specifichi concretamente la distinzione di base fra l'aspetto di compiutezza del PP e quello di aoristicità del PR, concentrandoci in particolare sul primo. Ci riferiamo alla lingua contemporanea, pur tenendo presente la difficoltà, particolarmente sensibile per l'italiano, di escludere usi arcaici che restano sempre disponibili come scelte stilistiche. Va però notato che il valore dei tempi del passato era notevolmente diverso nell'italiano antico: si veda Ambrosini 1961, Stussi 1961, Ageno 1964.

Il PP è un tempo bifronte che, come del resto il Perfetto latino, può valere, per la concordanza dei tempi (la consecutio temporum della grammatica latina) come un tempo principale (per usare la tradizionale terminologia scolastica): ho visto quello 
che fai, come vedo quello che fai, o come un tempo secondario o storico: ho visto quello che facevi, come vidi quello che facevi.

Ci sembra di poter distinguere i seguenti valori:

(a) Risultativo. Questo uso mette in luce l'aspetto di compiutezza del PP, cioè il punto finale del processo, mentre il PR indica l'azione in sé, a prescindere dal suo compimento. Il PP può avere un valore parafrasabile con un Presente (a volte designato come 'risultativo'), che indica uno stato conseguente all'azione, particolarmente con verbi momentanei, ingressivi, terminativi, ecc.: eccomi, sono arrivato ("sono qui"), è partito ("non è più qui"), me lo sono dimenticato ("non me lo ricordo"), ho capito ("mi è chiaro"), ho finito ("smetto"), si è raffreddato ("è freddo"), mi sono preso un raffreddore ("ho il raffreddore"), mi sono innamorato di lei ("la amo"), $\grave{e}$ morto ("non vive più"), ecc. Si noti che in casi come è morto si può essere incerti se si tratti di un PP o di una copula seguita da una forma aggettivale.

Con verbi durativi o stativi il PP situa l'azione al passato. Se mi sono innamorato di lei vuol dire "la amo", la ho amata sembra voler dire che "non la amo più". Si noti anche la differenza fra l'ho amata per un anno e l'amo da un anno: l'inglese $I$ have loved her for a year ha la struttura esterna della prima frase, ma il significato della seconda.

Naturalmente in contesti o co-testi opportuni anche nei casi citati il PP può riferirsi al passato (per es.: è arrivato il primo dell'anno ed è ripartito dopo due giorni), e occorre allora precisare come il suo valore si differenzia da quello del PR (arrivò il primo dell'anno e riparti dopo due giorni). Nella scelta fra PP e PR possono entrare in gioco considerazioni diverse.

(b) Pertinente. Una pare essere la pertinenza (che si trova nel PP, manca nel PR) rispetto alla situazione attuale: gli ho prestato un libro il 3 gennaio 1990 (e non me l'ha ancora restituito), vs gli prestai un libro il 3 gennaio 1990 (me lo restitui dopo una settimana, e da allora non l'ho più visto).

(c) Inclusivo. Un'altra pare essere il situarsi dell'azione (per il PP e non per il PR) entro lo stesso ambito cronologico del momento dell'enunciazione: negli ultimi cinque anni ho cambiato casa tre volte vs nel 1990 cambiai casa tre volte.

(d) Indefinito. Un'altra pare considerare l'azione compiuta bensì, ma spostata in un periodo anteriore indefinito, piuttosto che concentrata in un punto identificabile del passato; il PR invece si riferisce a un momento o a un'occasione nel passato in cui l'azione appare come compressa e precisamente localizzata: ho passato vari periodi a Parigi (spaziati in un passato indeterminato, senza riguardo alla loro durata o lontananza nel tempo), ma nel 1990 passai a Parigi le vacanze di Pasqua (un periodo 
definito, visto come un'unità, indipendentamente dal fatto che sia stato lungo o breve, e che si sia verificato molto o poco tempo fa).

(e) Recente. Un'altra ancora pare riferirsi alla distanza cronologica dal momento dell'enunciazione, minore per il PP che per il PR, quasi a giustificare la designazione di 'prossimo' e 'remoto': l'ho visto cinque minuti fa, ma lo vidi cinque anni fa.

Già a metà del Quattrocento Leon Battista Alberti (1964, p. 49) nella Grammatichetta illustra la differenza fra PP e PR con: Ieri fui ad Ostia: oggi sono stato a Tibuli; e nelle Prose (1525) il Bembo (1966, p. 244) osserva: »Ora tra queste due usanze di dire, Io feci e Io ho fatto, altra differenza non mostra che vi sia, se non questa: che l'una più propriamente si dà al passato di lungo tempo, e questa è $I o$ feci, e l'altra al passato di poco. Chè se io volessi dire d'aver scritti alcuni fogli, che io testé avessi forniti di scrivere, io direi Io gli ho scritti, e non direi Io gli scrissi. E se io questo volessi dire d'altri, che io di lungo tempo avessi scritti, direi lo gli scrissi diece anni sono, e non direi Io gli ho scritti«.

Nell'insegnamento scolastico si diceva tradizionalmente che il PP era adatto per gli eventi avvenuti entro la giornata in corso, e il PR per quelli precedenti. La norma si trova nella Grammaire générale et raisonnée di Port-Royal (1660, p. 104): »j'écrivis, je fis, $j$ 'allay, je disnay, \&c. Ce qui ne se dit proprement que d'un temps qui soit au moins éloigné d'un jour de celuy auquel nous parlons. Car on dit bien par example: j'écrivis hier, mais non pas, j'écrivis ce matin, ni j'éscrivis cette nuit; au lieu dequoy il faut dire, $j$ 'ay escrit ce matin, $j$ 'ay escrit cette nuit \&c«. A questo segue un commento interessante: »Nostre langue est si exacte dans la propriété des expressions, qu'elle ne souffre aucune exception en cecy, quoy que les Espagnols \& les Italiens confondent quelquefois ces deux preterits, les prenant l'un pour l'autre«.

Skubic (1967; 1970, p. 383) osserva che la distanza cronologica dell'evento, con l'opposizione fra un passato lontano (espresso dal PR), e uno recente (espresso dal PP) aveva pochissimo peso nel toscano antico: pare si sia diffusa nel Quattrocento e imposta nel Cinquecento. Essa diventerà l'elemento principale di distinzione fra $\mathrm{PP} \mathrm{e}$ PR nelle regole dei grammatici, e si imporrà sempre più anche nella lingua letteraria. Col generalizzarsi del PP il limite si è venuto peraltro spostando all'indietro, verso il passato, anche a Firenze; ma nelle parlate toscane rustiche esso corrisponde ancora a quello che troviamo nell' Alberti, che segna la separazione fra ieri (col PR) e oggi (col PP).

8. Non è ben chiaro fino a che punto i cinque fattori che abbiamo elencato siano necessari e sufficienti a caratterizzare l'uso del PP rispetto al PR. Si tratta di considerazioni semantiche e pragmatiche che non tanto derivano necessariamente dalla distinzione di base fra valenze aspettuali di compiutezza (per il PP) o di aoristicità (per il PR), quanto ad esse possono accompagnarsi. Questi cinque fattori non si escludono l'uno con l'altro, ma possono valere simultaneamente per spiegare un dato singolo 
esempio. Il motivo per distinguerli è la possibilità che si presentino dei casi in cui uno solo di essi, senza gli altri, sia l'elemento determinante per la scelta del PP o del PR.

Vanno tenute presenti due difficoltà. Una è costituita dal fatto che la scelta del tempo dipende di solito dal punto di vista assunto dal parlante, più che da dati esterni obiettivi, relativi al contesto extralinguistico. L'altra è che $i$ valori in questione possono entrare in conflitto. Per esempio, se ci riferiamo a un articolo scritto nel 1990 , senza indicarne la pertinenza rispetto al momento dell'enunciazione, o senza mettere in luce il carattere compiuto del processo, possiamo dire, ricorrendo al valore aoristico del PR: Questo saggio fu scritto nel 1990. Ma, pur senza cambiare per nulla l'interpretazione aspettiva della frase, possiamo ritenere il PR inappropriato alla vicinanza cronologica dell'evento, ed esprimere quest'ultima con il PP: Questo saggio è stato scritto nel 1990. In questo caso la scelta del tempo dipende non tanto da un'interpretazione diversa dell'azione quanto dalla scelta di far prevalere l'uno o l'altro dei due fattori che si riconoscono, comunque, compresenti nella caratterizzazione dell'evento.

Skubic $(1965 ; 1967 ; 1970 ; 1978)$ ricorre a tre opposizioni: la prima, fondamentale, fra valore di 'Aoristo' (per il PR) e di 'Perfetto Presente' (per il PP), che pare coprire i nostri tre fattori (a), (b), (c); la seconda fra 'Azione Punto' (per il PR) e azione non puntualizzata (per il PP), che corrisponde al nostro (d); e la terza fra azione remota (per il PR) e recente (per il PP), che corrisponde al nostro (e).

L'opposizione primaria, fra valore di aoristo (per il PR) e di perfetto presente (per il PP), caratteristica del toscano antico, è ancora viva in quello contemporaneo (ma più nella parlata rustica che a Firenze) e viene illustrata da Skubic (1970, p. 361) con la frase seguente, pronunciata da un commerciante di Poppi (paese del Casentino, in provincia di Arezzo) nel 1956: Questa macchina [nella quale siedono gli interlocutori], io l'ho comprata nel 1953 per un milione: due mesi dopo usci fuori un altro tipo per sole ottocento mila [lire]. Di questo contrasto può render conto il nostro fattore (b):pertinente.

Bertinetto (1986, pp. 414-428) specifica tre tipi di usi deittici (cioè rapportati al momento dell'enunciazione) per il PP: (1) persistenza del risultato: Giorgio è arrivato, che corrisponde al nostro (a): risultativo, e ho comprato questa macchina un paio di lustri fa (e ce l'ho ancora), che corrisponde al nostro (b): pertinente; (2) esperienziale o esistenziale: sei mai stato in Francia?, che pare assimilabile al nostro (d): indefinito; (3) inclusivo: negli ultimi due mesi, Bernardo ha vissuto in condizioni molto disagevoli, che corrisponde al nostro (c): inclusivo; e cinque tipi di non-deittici (cioè con punti di riferimento sganciati dal momento dell'enunciazione): (4) anteriorità nel futuro: gli dirai che sei venuto; (5) anteriorità nel passato (equivalente al trapassato prossimo): la casa è crollata dopo che tu sei uscito; (6) atemporale: una persona che ha studiato non deve comportarsi cosi; (7) abituale: tutte le volte che lo incontro mi parla dell'ultimo libro che ha letto; (8) imminenziale: prima di sera siamo arrivati, o domani ho finito. $\mathrm{Ci}$ pare che i casi (4)-(7) possano rientrare nel nostro (d): indefinito; (8) esemplifica un interessante modo di vivacizzare l'esposizione, pre- 
sentando il futuro come già arrivato, appena compiuto sotto i nostri occhi, in maniera che ci pare corrispondere ai nostri (a) e (d).

La ricerca di esempi in cui sia possibile il PP e non il PR, o viceversa, si scontra con qualche difficoltà. Un elemento come la distanza temporale (fattore (e): recente) dovrebbe essere abbastanza obiettivamente misurabile; e di fatto, durante un seminario alla Scuola Normale di Pisa nel 1976, con la partecipazione anche di parlanti toscani, si rivelò difficile ottenere delle frasi al PR che si riferissero ad avvenimenti della stessa giornata. Non è però che esse siano impossibili. Bertinetto (1986, p. 428) cita: Venendo qui, vidi Gennaro che andava alla stazione: sarà stato cinque minuti fa, precisando però che un esempio di questo genere si può avere sin certe varietà di italiano, che pure utilizzano correntemente « sia il PP sia il PR. La formulazione fa pensare che non si tratti di toscano. Del resto la reazione dei parlanti toscani a cui ci siamo rivolti, riguardo ai cambiamenti di significato implicati dall'alternanza fra PP e $P R$, è spesso incerta.

Più definiti sembrano $i$ casi in cui si hanno specificazioni avverbiali che siano compatibili con uno dei valori aspettuali (compiutezza o aoristicità) ma non con l'altro. Per esempio, Bertinetto (1986, pp. 195, 203, 412) nota che "da X tempo" si riferisce al punto terminale del processo, ed è compatibile con l'aspetto compiuto del PP, ma non con quello aoristico del PR: Mario è arrivato da almeno due ore, ma non *Mario arrivò da almeno due ore. Così con già e non ancora (p. 196): ha già letto (*lesse) il libro che gli hai prestato; non è ancora uscito (*usci). Con avverbi come finalmente (p. 227) il valore di compiutezza del PP si riferisce al momento finale dell'azione: finalmente ha mangiato ("ha finito di mangiare"), mentre l'aspetto aoristico del PR introduce un valore ingressivo: finalmente mangiò ("si mise a mangiare, decise di smettere il digiuno").

Bertinetto (1986, pp. 428-429) nota anche che il PR, essendo sempre deittico (indicando cioè il passato rispetto al momento dell'enunciazione), non può costituire un'alternativa, sia pure con significato diverso, per il PP non deittico. Al posto di vengo dopo che ho mangiato non possiamo avere *vengo dopo che mangiai.

Altri casi possono essere più incerti. Bertinetto (1986, p. 431) osserva che vissi a lungo in quel posto è accettabile solo se il proceso è terminato, cioè se in quel posto non ci vivo più; ma Toti Scialoja (1985, p. 431) scrive: „C'era un geco presso Amalfi / all'ingresso del suo speco / ridacchiava sotto i baffi / e mi disse: 'Pissi, pissi. / È cent'anni che qui vissi'. / Poi mi disse: 'Passi! passi! / Non vedrà che sassi e sassi' «.

9. Abbiamo parlato della presenza di toscani nel citato seminario alla Normale. Questo è pertinente perché, come è noto, l'uso del PP e del PR nel parlato spontaneo differisce regionalmente. L'immagine tradizionale è che nell'Italia settentrionale non si usi affatto il PR (in molti dialetti addirittura scomparso, non più disponibile nella morfologia verbale); che la distinzione fra PP e PR sia viva in Toscana e nell'Italia centrale; e che nell'Italia meridionale, e particolarmente in Sicilia, prevalga il PR. 
Borgato (1976, p 95) ritiene che l'uso toscano riveli non una distinzione sistematica, assente sia al Nord sia al Sud, bensì un'incertezza fra il PP settentrionale e il PR meridionale: »Nell'Italia centrale la situazione è fluida, ma per quanto alcuni abbiano voluto vedere, nelle parlate della Toscana, dell'Umbria e in parte delle Marche e del Lazio, nelle quali vengono impiegate entrambe le forme, una sopravvivenza di opposizione aspettuale e/o di locazione temporale tra PP e PR, penso sia più rispondente alla realtà parlare invece di influenze diverse in contrasto tra loro«.

L'italiano letterario, ovviamente, usa sia il PP sia il PR, ma tale uso è meno univoco e definito che in altre lingue, come per es. l'inglese, presumibilmente anche in rapporto alla diversità rispetto al parlato. Per un settentrionale la presenza del PR è già di per sé un indice di letterarietà, e ci sono forme di italiano scritto più vicine al parlato (per es. la corrispondenza con i familiari, particolarmente su argomenti privati e domestici) in cui un settentrionale, anche colto, tenderà a non usare il $\mathrm{PR}$, in quanto esso indicherebbe un salto di registro ("ti scrivo come se si trattasse non di una conversazione ma di un testo letterario"). Di fatto l'uso caratteristico del PR nell' italiano letterario si trova all'interno di un racconto, per indicare fatti collegati in una successione cronologica.

Se e come l'uso letterario si differenzi dal parlato toscano, non sapremmo dire con precisione, e non abbiamo presenti analisi sistematiche sull'argomento. Dagli studi disponibili risulta che l'uso colloquiale toscano presenta il PR anche in certi casi in cui la lingua letteraria ricorrerebbe probabilmente al PP. Per esempio, per eventi vicini: tu venisti sabato sera, vero?; e ieri, noi si andò a pranzare da Gigi (Skubic 1965, p. 86); I' Torino vinse un'a zero com'ho già detto; e Un ti dico come successe (Stammerjohann 1970, pp. 306-307).

D'altra parte, di fronte a un testo come Cuore, scritto dal ligure De Amicis anche come modello di lingua, si ha la sensazione che ci sia un eccesso ipercorretto di PR: bisogna leggere varie pagine di questo diario, prima di trovare un PP. Giorgio Pasquali (1951, p. 190) osserva che De Amicis »apparteneva a quei settentrionali per i quali il passato remoto è un'eleganza estranea alla lingua comune, e che quindi ne abusano, anzi ne usano a sproposito《, citando appunto l'inizio di Cuore: »Oggi primo giorno di scuola. Passarono come un sogno quei tre mesi di vacanza in campagna! Mia madre mi condusse questa mattina alla sezione Baretti«.

Il problema è che si ha un processo di espansione del PP rispetto al PR, già dal Medioevo nell'Italia settentrionale (dove si è arrivati alla scomparsa del PR), e dal Rinascimento, in maniera più limitata, anche in Toscana. Il fatto che il PP possa assumere le funzioni del PR provoca una situazione incerta e confusa nella lingua letteraria (che si riflette anche nelle insufficienze della tradizione grammaticale su questo punto).

10. Come si è accennato, con un testo inglese non sarebbe possibile sostituire tutte le ricorrenze di Simple Past con un Present Perfect senza introdurre casi di 
agrammaticalità, o cambiamenti di significato. Potrebbe essere interessante (abbiamo fatto qualche prova, di cui parleremo altrove, accostando testi originali e in traduzione; si vedano considerazioni contrastive in Wandruszka 1968, Saronne 1971, Ondraček 1971, Borgato 1976, Miklič 1983) fare un confronto preciso fra il sistema inglese e quello italiano. Le differenze sono sufficienti a creare difficoltà per i nostri alievi anglofoni (e viceversa per gli italiani che si servono dell'inglese).

L'inglese distingue dunque il Present Perfect (I have written a letter "ho scritto una lettera") dal Simple Past (I wrote a letter "scrissi/scrivevo/ho scritto una lettera"), e dalla forma progressiva (I was writing a letter "scrivevo/stavo scrivendo una lettera").

Il Simple Past si riferisce, implicitamente o esplicitamente, al momento specifico, alla singola occasione in cui il fatto si è verificato. Il Present Perfect lascia indeterminato il momento dell'evento, entro un periodo che arriva al momento dell'enunciazione (uso inclusivo).

Il motivo per cui in inglese la presenza di una indicazione temporale precisa richiede il Simple Past e esclude il Present Perfect si può spiegare ricordando le considerazioni di Reichenbach. Il Simple Past pone un punto di riferimento nel passato, e questo è ulteriormente specificabile con un dato più preciso, mentre il Present Perfect ha come punto di riferimento il momento dell'enunciazione, e non è perciò compatibile con l'indicazione di un diverso punto di riferimento nel passato (Guenthner 1977, p. 96; Åqvist 1978).

Questo rivela subito delle differenze rispetto all'italiano. In inglese non c'è difficoltà a dire I saw him a moment ago "l'ho visto un momento fa" (il nostro caso (e): recente), mentre *I have seen him a moment ago sarebbe impossibile. Così in italiano possiamo avere Shakespeare ha scritto, con l'Amleto, il dramma più famoso della letteratura inglese, in corrispondenza del nostro caso (d): indefinito, in quanto non ci riferiamo al momento esatto in cui è stato scritto, o anche in base a (b): pertinente, se riportiamo l'Amleto direttamente al nostro ragionamento, o a (c): inclusivo, se riteniamo che la letteratura inglese sia ancora in corso. In Inglese invece dovremo avere Shakespeare wrote, with Hamlet, the most famous play in English literature, mentre *has written suonerebbe strano, perchè parrebbe indicare che l'autore è ancora vivo e in grado di produrre altre opere. Quindi anche condizioni che sembrano formulabili in maniera analoga per le due lingue funzionano in realtà diversamente.

Del resto anche in inglese l'uso è passibile di incertezze e disaccordi (McCoard 1978), sia pure meno vistosi che in italiano. Comrie (1976, pp. 60-61) dissente da Chomsky e McCawley nella valutazione di Princeton has been visited by Einstein come accettabile in quanto Princeton, soggetto, esiste ancora (mentre Einstein has visited Princeton è anomalo in quanto si sa che il soggetto, Einstein, è morto). Un esempio analogo è Newton has explained the movements of the moon, che pare accettabile se si sta parlando della luna piuttosto che di Newton; e infatti la frase diventa 
più normale se diciamo the movements of the moon have been explained by Newton (Guenthner 1977, p. 92).

La differenza fra Simple Past e Present Perfect è tale che essa da sola è sufficiente a cambiare il valore di verità di una frase inglese. Una affermazione può essere falsa col Simple Past e vera col Present Perfect, come si può vedere da due battute consecutive (riportate sul Times del 24 luglio 1963) in un famoso processo in cui furono coinvolti vari personaggi altolocati. »Did you have intercourse with the defendant?« viene chiesto a una signorina, e la risposta è: »No«. La domanda successiva è: »Have you had intercourse with the defendant? «, e questa volta la risposta è »Yes«. Non si tratterà dell'inglese più scorrevole e familiare, ma l'esempio è autentico, ed esplicito. In italiano sembrerebbe necessario che il contrasto fra le due domande si appoggiasse su specificazioni avverbiali oltre che sull'uso dei tempi: »Ebbe allora rapporti con l'imputato? « e »Ha mai avuto rapporti con l'imputato?«(Lepschy 1988, p. 130).

\section{Riferimenti}

(a) Per il tempo e l'aspetto:

ÅQVIST, Lennart, 1978, On the Distinction between the Present Perfect and the Simple Past in English, in Rohrer, Christian, ed., Papers on Tense, Aspect and Verb Classification, Narr, Tübingen, pp. 37-48.

BENVENISTE, Émile, 1966, Problèmes de liguistique générale, Gallimard, Paris (tr. ital. Problemi di linguistica generale, il Saggiatore, Milano 1971).

_, 1974, Problèmes de liguistique générale II, Gallimard, Paris (tr. ital. Problemi di linguistica generale II, il Saggiatore, Milano 1985).

COMRIE, Bernard, 1976, Aspect. An Introduction to the Study of Verbal Aspect and Related Problems, Cambridge U.P., Cambridge.

,- 1985 , Tense, Cambridge U.P., Cambridge.

COSERIU, Eugenio, 1976, Das romanische Verbalsystem, a cura di Hansbert Bertsch, Narr, Tübingen.

DAHL, Östen, 1985, Tense and Aspect Systems, Blackwell, Oxford.

DIETRICH, Wolf, 1973, Der periphrastische Verbalaspekt in den romanischen Sprachen (ZRPh, Bh. 140), Niemeyer, Tübingen.

GRETSCH, Nic., 1828, Grammaire raisonnée de la langue russe, tr. Ch.Ph.Reiff. Imprimerie de Nicolas Gretsch, Saint-Petersbourg.

GUENTHNER, F., 1977, Remarks on the Present Perfect in English, in Rohrer, Christian, ed., On the Logical Analysis of Tense and Aspect, Narr, Tübingen, pp. 8398.

HOLT, Jens, 1943, Études d'aspect (Acta Jutlandica 15, 2), Munksgaard, København. 
HOPPER, Paul J., ed., 1982, Tense-Aspect. Between Semantics and Pragmatics, Benjamins, Amsterdam.

JAKOBSON, Roman, 1971, Shifters, Verbal Categories, and the Russian Verb, Selected Writings, II: Word and Language, Mouton, The Hague, pp. 130-147.

KNOBLOCH, Johann, 1961-1986, Sprachwissenschaftliches Wörterbuch, I, Winter, Heidelberg, pp. 76-78, 172-80.

KURYLOWICZ, Jerzy, 1966, L'évolution des catégories grammaticales, in Problèmes du langage (Collection Diogène), Gallimard, Paris, pp. 54-71 (tr. ital. Evoluzione delle categorie grammaticali, in I problemi attuali della linguistica, Bompiani, Milano 1968, pp. 65-84).

— 1977 , Problèmes de linguistique indo-européenne (Prace Jezykoznawcze 90), Polska Akademia Nauk, Wroclaw, pp. 53-89.

LO CASCIO, Vincenzo, VET, Co, eds, 1986, Temporal Structure in Sentence and Discourse, Foris, Dordrecht.

LYONS, John, 1977, Semantics, Cambridge U.P., Cambridge, pp. 636-724.

MATTHEWS, Peter, 1990, La linguistica greco-latina, in Lepschy, Giulio, a cura di, Storia della linguistica, I, il Mulino, Bologna, pp. 187-310.

McCOARD, Robert W., 1978, The English Perfect: Tense-Choice and Pragmatic Inferences, North-Holland, Amsterdam.

NESTOR-SCHNURMANN, J., 1884, The Russian Manual, Allen, London.

RAMAT, Paolo, 1983, Habere, + PPP: una nota, in Scritti linguistici in onore di Giovan Battista Pellegrini, a cura di Paola Benincà et al., Pacini, Pisa, pp. 14531462.

REICHENBACH, Hans, 1947, Elements of Symbolic Logic, Macmillan, New York.

$\mathrm{REIFF}, \mathrm{Ch} . \mathrm{Ph} ., 1851$, Grammaire russe précédée d'une introduction sur la langue slavonne, Saint-Petersbourg, \& Th. Barrois, Paris.

—, 1857, English-Russian Grammar, Second edition, Maisonneuve, Paris.

WEINRICH, Harald, $1971^{2}$, Tempus. Besprochene und erzählte Welt, Kohlhammer, Stuttgart (tr. ital. Tempus. Le funzioni dei tempi nel testo, il Mulino, Bologna 1978). 
(b) per l'italiano:

AGENO BRAMBILLA, Franca, 1964 Il verbo nell'italiano antico. Ricerche di sintassi, Ricciardi, Milano-Napoli.

ALBERTI, Leon Battista, 1964, La prima grammatica della lingua volgare, a cura di Cecil Grayson, Commissione per i testi di lingua, Bologna.

AMBROSINI, Riccardo, 1961, L'uso dei tempi storici nell'italiano antico, L'Italia Dialettale, 24, pp. 13-124.

BEMBO, Pietro, 1966, Prose e rime, a cura di Carlo Dionisotti, UTET, Torino.

BERTINETTO, Pier Marco, 1986, Tempo, aspetto e azione nel verbo italiano. Il sistema dell'indicativo, Accademia della Crusca, Firenze.

BLÜCHER, Kolbjørn, 1974, Studio sulle forme ho cantato, cantai, cantavo, stavo cantando, Universitetsforlaget, Bergen.

BORGATO, Gianluigi, 1976, Aspetto verbale e Aktionsart in italiano e in tedesco, Lingua e contesto, 3, pp. 95-197.

[LANCELOT, Claude, ARNAULD, Antoine], 1660, Grammaire générale et raisonnée, Pierre le Petit, Paris (tr. it. Grammatica e logica di Port-Royal, a cura di Raffaele Simone, Ubaldini, Roma 1969).

LEPSCHY, Anna Laura \& Giulio, $1988^{2}$, The Italian Language Today, Unwin Hyman, London.

LEPSCHY, Giulio, 1985, recensione di Tumler 1980, Romance Philology, 39:2, pp. 250-253.

$\longrightarrow, 1988$, recensione di Bertinetto 1986, Journal of Pragmatics, 12:1, pp. 128-130.

LUCCHESI, Valerio, 1971, Fra grammatica e vocabolario. Studio sull'»aspetto« del verbo italiano, Studi di Grammatica Italiana, 1, pp. 179-269.

MIKLIČ, Tjaša, 1983, L'opposizione italiana perfetto vs imperfetto e l'opposizione slovena dovršnost vs nedovršnost nella verbalizzazione delle azioni passate, Linguistica, 23, pp. 53-123.

_ 1991 , Presenza e valori del passato remoto in riassunti di opere letterarie, Linguistica, 31, pp. 249-258.

ONDRÁČEK, Jaroslav, 1971, Some Notes on the Difference between the Present Perfect and the Simple Past in English and in Italian, Sborník Prací Filosofičké Fakulty Brnenské University, A-19, pp. 167-178.

PASQUALI, Giorgio, 1951, Stravaganze quarte e supreme, Neri Pozza, Venezia.

ŠABRŠULA, Jan, 1961, Les équivalents de l'aspect slave en italien (Étude comparative), Philologica Pragensia, 4, pp. 147-160.

SARONNE, Edgardo T., 1970, La questione dell'aspetto verbale in italiano, Lingua e Stile, 5, pp. 271-281.

- 1971, Considerations on the Category of Aspect in Russian and in Italian, Lingua e Stile, 6, pp. 51-66. 
SAVIĆ, Momčilo D., 1979, L'uso dei tempi passati nei quotidiani pubblicati nelle lingue romanze con particolare riguardo all'italiano, Linguistica, 19, pp. 171197.

SKUBIC, Mitja, 1965, Il preterito nel toscano parlato, Linguistica, 7, pp. 85-90. 1967, La sorte del preterito nel Bembo e in altri Cinquecentisti, Lingua Nostra, 28, pp. 19-22.

— 1970 , Contributi alla storia del preterito nell'italiano, Razprave (Slovenska Akademija Znanosti in Umetnosti, Razred za filološke in literarne vede), 7, pp. 343-400.

, 1978, Sur les valeurs des temps du passé dans les langues romanes, Linguistica, 17, pp. 143-159.

STAMMERJOHANN, Harro, 1970, Strukturen der Rede, Studi di Filologia Italiana, 28, pp. 296-397.

STUSSI, Alfredo, 1961, Imperfetto e passato remoto nella prosa volgare del Quattrocento, L'Italia Dialettale, 24, pp. 125-133.

TEKAVČIĆ, Pavao, 1970, Saggio di un'analisi del sistema verbale italiano, Lingua e Stile, 5, pp. 1-23.

TUMLER, Tilman, 1980, Der Tempusgebrauch der Vergangenheit in der modernen italienischen Prosa, (Wiener romanistische Arbeiten 12), Braumüller, Wien.

WANDRUSZKA, Mario, 1968, L'aspect verbal, problème de traduction, Travaux de Linguistique et de Littérature, 6:1, pp. 113-123.

\section{Povzetek}

\section{GLAGOLSKE OBLIKE ZA PRETEKLOST}

Avtorja pretresata véliki in tolikokrat obdelani problem vrednosti glagolskih oblik za preteklost $\mathrm{v}$ italijanščini, imperfekta, enostavnega in sestavljenega preteklika, torej tistih, katerih vrednosti se prepletajo $z$ glagolskim vidom $v$ slovanskih jezikib. Začenjata s kronologijo pojava, ki mu je ravno slovansko jezikoslovje dalo ime "glagolski vid", "aspekt"; opozarjata, da je vid pravzaprav napačen prevod: eidos pomeni v grščini 'vrsto', 'kategorijo'. Razmejujeta aspekt od (na nemških univerzah zraslega izraza) Aktionsart. Tehtata, nadalje, ustreznost definicij za te glagolske paradigme $\mathrm{v}$ italijanskih slovnicah od najstarejše, neizdane, ki je delo italijanskega renesančnika Leona Battiste Albertija pa vse do današnjih dni.

Študija opozarja, da je bila raba v starih toskanskih besedilih dokaj drugačna, arhaična raba pa đa je še zmeraj mogoča, seveda kot stilistično sredstvo. Zelo natančno je analizirana raba enostavnega preteklika proti sestavljenemu, posebej še $\mathrm{v}$ Toskani, opozarja pa se tudi na primere, kjer je raba ene ali druge paradigme izključena: v stavku Mario è arrivato da almeno due ore 'Mario je prišel vsaj že pred dvema urama' enostavni preterit ni uporabljiv; so pa stavčne dvojnice, kjer izbira paradigme spremeni pomen: Finalmente ha mangiato/Finalmente mangiò: $\mathrm{v}$ drugem primeru gre za ingresivni aspekt 'končno se je spravil $\mathrm{k}$ jedi/sklenil je, da bo nehal s postom'.

Študijo končuje kratka, zgoščena primerjava med sistemom v italijanščini in angleščini; opozarja se na negotovost italijanskega govorca (velja pa seveda tudi obratno), ko skuša glagolski sistem svojega jezika uporabiti $v$ angleščini. 